\title{
Adopción de redes sociales por revistas científicas de ciencias sociales
}

\section{Adoption of social media by social sciences scientific journals}

\author{
Carlos Arcila-Calderón; Mabel Calderín-Cruz; Patricia Sánchez-Holgado
}

Cómo citar este artículo:

Arcila-Calderón, Carlos; Calderín-Cruz, Mabel; Sánchez-Holgado, Patricia (2019). “Adopción de redes sociales por revistas científicas de ciencias sociales". El profesional de la información, v. 28, n. 1, e280105.

https://doi.org//10.3145/epi.2019.ene.05

Artículo recibido el 11-10-2018

Aceptación definitiva: 14-12-2018

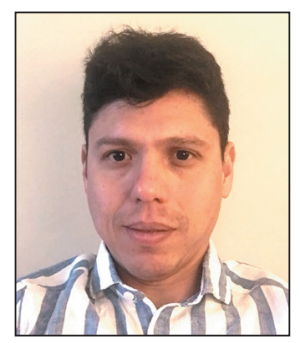

\section{Carlos Arcila-Calderón http://orcid.org/0000-0002-2636-2849}

Universidad de Salamanca Facultad de Ciencias Sociales Campus Miguel de Unamuno, Edificio FES Av. Francisco Tomás y Valiente, $s / n$. 37071 Salamanca, España carcila@usal.es

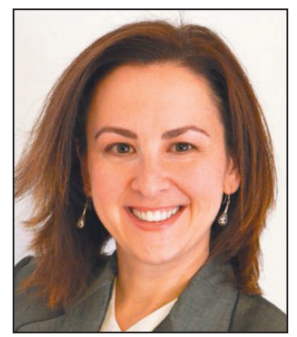

\section{Patricia Sánchez-Holgado} http://orcid.org/0000-0002-6253-7087

Universidad de Salamanca Facultad de Ciencias Sociales Campus Miguel de Unamuno, Edificio FES Av. Francisco Tomás y Valiente, $\mathrm{s} / \mathrm{n}$. 37071 Salamanca, España patriciasanc@usal.es

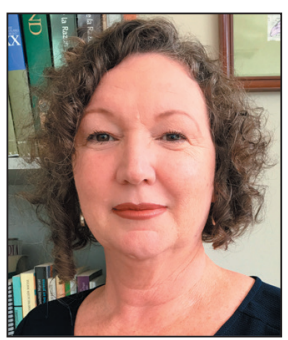

Mabel Calderín-Cruz http://orcid.org/0000-0002-7750-4608

Centro Cultural Padre Carlos Guillermo Plaza

Universidad Católica Andrés Bello. Moltabán, Caracas, Venezuela mcalderi@ucab.edu.ve

\section{Resumen}

La expansión de las redes sociales obliga a la comunicación de la ciencia y a las revistas científicas a adaptarse al nuevo entorno. Estudios anteriores y modelos teóricos como Utaut y Utaut2, han probado que existe una relación entre la percepción de desempeño futuro de la tecnología (Expectativa de rendimiento), la dificultad de uso (Expectativa de esfuerzo) y la Influencia social a la que el individuo está sometido, sobre el uso real de dicha tecnología, pero aún existe poca investigación acerca de los mecanismos que causan dicho efecto. Partiendo de Utaut, la hipótesis planteada es que existe un efecto condicional indirecto de la Expectativa de rendimiento, la Expectativa de esfuerzo y la Influencia social, sobre el uso real de redes sociales en las revistas científicas, donde dicho efecto es mediado por la intención de uso y moderado por el género y la edad. Se realizó una encuesta a 300 editores de ciencias sociales del ranking Google Scholar. Confirmando parcialmente el modelo Utaut, los resultados de la mediación moderada evidencian que la Expectativa de rendimiento y la Influencia social en los editores inciden en el uso real de Facebook y Twitter, a través de la intención de uso, pero no sucede así en el caso de ResearchGate, Academia y LinkedIn. No hay evidencia clara de que la edad y el género moderen los predictores estudiados.

\section{Financiación}

Centro Cultural Padre Carlos Guillermo Plaza SJ de la Universidad Católica Andrés Bello, Venezuela.

Agradecimiento

A Andrea Urbina por su participación en la recogida de datos. 


\title{
Palabras clave
}

Adopción de tecnologías; Uso de tecnologías; User acceptance and use of technology; Utaut; Redes sociales; Medios sociales; Editores; Revistas científicas; Ciencias sociales; Twitter; Facebook; LinkedIn; ResearchGate; Academia.edu.

\begin{abstract}
The expansion of social media forces the communication of science and scientific journals to adapt to this new environment. Previous studies and theoretical models such as Utaut and Utaut2 have proven that there is a relationship between the perception of future performance of technology (performance expectancy), the difficulty of use (effort expectancy) and the social influence to which the individual is subject, on the real use of technology, but there is still scarce research about the mechanisms that cause this effect. Starting with Utaut, the hypothesis is that there is an indirect conditional effect of the performance expectancy, the effort expectancy and social influence, over the actual use of social media in scientific journals, where the effect is driven through the intention of use and the size of the effect is moderated by gender and age. A survey was carried out on 300 editors of the top 20 in Social Sciences of the Google Scholar ranking. Confirming partially Utaut, the results of moderated mediation show that performance expectancy and social influence of editors affect the use of Facebook and Twitter, through the intention to use, but it is not the case of ResearchGate, Academia and LinkedIn. There is not enough evidence about the moderation of age and gender.
\end{abstract}

\section{Keywords}

Adoption of technologies; Use of technologies; User acceptance and use of technology; Utaut; Social media; Editors; Scientific journals; Social Sciences; Twitter, Facebook; LinkedIn; ResearchGate; Academia.edu.

\section{Introducción}

El uso de las nuevas tecnologías de la información y la comunicación (TIC) ha cambiado las prácticas de comunicación científica (Gibbons et al., 1994) y las revistas científicas dentro del paradigma de la llamada ciencia 2.0 (Waldrop, 2008 ), o ciencia abierta (Neylon; Wu, 2008; García-Peñalvo, 2017).

De hecho, la ciencia abierta ha modificado la forma en que investigadores, editores y revistas hacen su trabajo en ciencias sociales (Borgman, 2007; Dutton, 2010), incluso aunque la adopción de las TIC pueda parecer lenta, comparada con otros campos como la Física (Gentil-Beccot et al., 2009).

Las revistas científicas son consideradas un instrumento principal en la comunicación de la ciencia (Castillo-Esparcia, 2012). En este contexto, aunque sigue habiendo resistencia al movimiento de acceso abierto (Schiermeier, 2017), algunos editores están dispuestos a adoptar redes sociales como medio de compartir la ciencia y sus resultados entre investigadores y públicos no especializados, migrando publicaciones tradicionales hacia interactivas. El nacimiento de este tipo de publicaciones académicas en ciencias sociales -debido a la adopción de las redes sociales- representa una clara innovación en la forma en que las revistas interactúan con sus autores y lectores.

Por otra parte, la difusión del conocimiento científico a través de las redes sociales acelera el alcance obtenido por los resultados de la investigación, dando más visibilidad a la ciencia y promoviendo el aprendizaje abierto. Los editores son actores clave en este proceso, debido a su gran influencia sobre las editoriales y asociaciones científicas. Como expertos locales, los editores son vitales en la domesticación de las TIC (Stewart, 2007), lo que hace relevante la comprensión de los factores que impulsan a estos agentes a adoptar los medios sociales en sus revistas y la forma en que estos factores directa o indirectamente afectan el uso real de las redes sociales en interacción con atributos personales como el sexo o la edad.

Algunos estudios anteriores han abordado las razones por las que los investigadores adoptan las redes sociales para comunicar sus resultados (Santana-Arroyo, 2011; Arcila-Calderón; Calderín-Cruz; Aguaded, 2015), sin embargo no existen estudios precedentes sobre los editores de revistas científicas desde una perspectiva teórica de adopción de innovaciones tecnológicas.

Este trabajo pretende llenar ese vacío analizando los factores que impulsan a los editores de revistas de ciencias sociales a adoptar los medios sociales. Sobre todo, busca comprender los mecanismos y condiciones bajo los cuales operan estas variables, es decir, valorar las relaciones de mediación para conocer los efectos indirectos de una variable sobre otra a través de una tercera y las relaciones de moderación. En las siguientes secciones ofrecemos un panorama de la investigación realizada y el marco teórico que apoya nuestras hipótesis. A continuación, describimos el método de esta investigación, un informe de las conclusiones, y finalmente presentamos una discusión. 


\section{Medios sociales y publicaciones científicas}

En los últimos años las revistas científicas han ido adoptando progresivamente tanto redes sociales generalistas (Twitter y Facebook), como académicas (ResearchGate y Academia); y profesionales (LinkedIn) (Procter et al., 2010; Ponte; Simon, 2011; Campos-Freire; Valencia, 2015; Campos-Freire; Rúas-Araújo, 2016).

Este hecho se explica por las múltiples interconexiones que los medios sociales producen entre los estudiosos, lo que permite compartir fácilmente datos y acelerar la colaboración científica (Murthy; Lewis, 2015). En este sentido, la adopción de estas innovaciones podría estar relacionada con la tasa de colaboración científica (Hara et al., 2003; Cummings; Kiesler, 2005; Rigby; Edler, 2005) o incluso el factor de impacto individual (Liao, 2011; Direito-Rebollal; Campos-Freire, 2016). Existe una extensa bibliografía sobre cómo los investigadores se han adaptado a las e-infraestructuras emergentes y a las TIC en ciencias sociales y humanidades en Estados Unidos (Barjak et al., 2010), Europa (Voss et al., 2010; Procter; Voss; Asgari-Targhi, 2013) y América Latina (Briceño; Arcila-Calderón; Said-Hung, 2012; Arcila-Calderón; Piñuel-Raigada; Calderín-Cruz, 2013; Briceño, 2014). Hay pruebas sólidas de que las actitudes hacia la e-social research son positivas (Meyer; Dutton, 2009) pero con un apoyo inicial limitado (Dutton; Meyer, 2009).

Específicamente, compartir los trabajos académicos en la web 2.0 ha sido considerado un reto (Acord; Harley, 2013), porque el uso de sitios como Twitter aumenta significativamente la difusión y el impacto de la investigación original (Bruns; Liang, 2012). Estudios similares también han abordado esta preocupación en el campo de la publicación académica examinando el papel de los repositorios abiertos que usan software DSpace o Eprints, el intercambio de documentos peer-to-peer (email, ResearchGate, etc.), o piratería (Sci-Hub, LibGen).

En países como España, estudios anteriores encontraron que las herramientas de la web 2.0 apenas fueron usadas por revistas de ciencias sociales (Oller-Alonso; Segarra-Saavedra; Plaza-Nogueira, 2012), incluso cuando esta innovación podría impactar positivamente en la comunicación científica en términos de marketing viral (Bellón-Rodríguez; Sixto-García, 2011). Sin embargo, no existen estudios comparativos o internacionales que expliquen la adopción de los medios sociales por revistas en ciencias sociales, por lo que nos preguntamos:

RQ1. ¿Hasta qué punto redes las sociales generalistas (Twitter o Facebook), académicas (ResearchGate o Academia), y profesionales, (Linkedln) son adoptadas por revistas científicas en ciencias sociales?

\section{Expectativa de rendimiento, Expectativa de esfuerzo e Influencia social}

El marco de difusión de la innovación se ha aplicado a las TIC en diferentes contextos como la adopción de medios sociales por los políticos (Quinlan et al., 2017) o por científicos (Arcila-Calderón; Calderín-Cruz; Aguaded, 2015). Ahora buscamos los factores que conforman la adopción de redes sociales en publicaciones académicas (utilizando sus editores como una fuente de confianza) y explorando los mecanismos y condiciones bajo los cuales operan. Existen varios modelos de aceptación de la tecnología que derivan del paradigma de difusión de innovaciones. Entre ellos, Utaut (User acceptance and use of technology o Teoría unificada de aceptación y uso de tecnología), propuesta por Venkatesh et al. (2003), que maneja variables como la expectativa de rendimiento, expectativa de esfuerzo, influencia social, condiciones facilitadas, entre otras; y el modelo Utaut2 (Venkatesh; Thong; Xu, 2012), que incorpora las variables de motivación hedónica, valor de precio, experiencia y hábito.

En este estudio seguimos un modelo simplificado de tres factores principales basado en Utaut (figura 1):

1) Expectativa de rendimiento: grado en que el uso de una tecnología proporcionará algún beneficio al usuario al realizar ciertas actividades;

2) Expectativa de esfuerzo: facilidad de utilización de una tecnología percibida por parte del usuario;

3) Influencia social: medida en que los usuarios perciben que personas cercanas consideran apropiado el uso de una tecnología.

El modelo establece que hay un efecto condicional indirecto de estas tres variables sobre el uso real de las redes sociales. Dicho efecto es mediado por la intención de uso y moderado por el género y la edad. En línea con Utaut y para probar estas afirmaciones, planteamos que:

H1. La Expectativa de rendimiento influye de manera positiva en la intención de uso de redes sociales por los

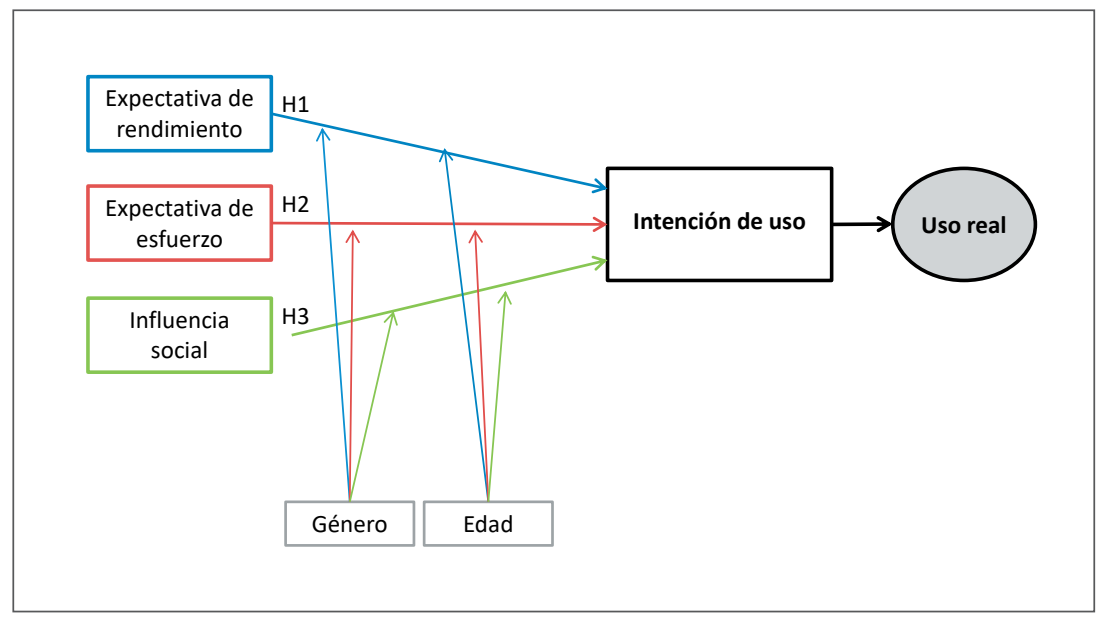

Figura 1. Modelo simplificado de tres factores principales de Utaut. 
editores, generando un efecto indirecto positivo en su uso real. Dicho efecto indirecto está moderado por el género y la edad, de manera que será más fuerte en los hombres y en los jóvenes.

H2. La Expectativa de esfuerzo influye negativamente en la intención de uso de redes sociales por los editores, generando un efecto indirecto negativo en su uso real. Este efecto indirecto está moderado por el género y la edad, siendo más fuerte en las mujeres y en las más mayores.

H3. La Influencia social de los editores influye positivamente en la intención de uso de redes sociales, lo que a su vez genera una influencia positiva en su uso real. El efecto indirecto es moderado significativamente por el género y la edad, de modo que será más fuerte en las mujeres y en las personas de más edad.

\section{Método}

\subsection{Participantes y procedimientos}

Se calculó el tamaño muestral mínimo requerido para contrastar las hipótesis formuladas teniendo en cuenta que se realizaría un análisis de regresión lineal con tres predictores basado en mínimos cuadrados ordinarios. La estimación se hizo en G*Power 3.1 (Faul et al., 2009), utilizando como parámetros los tamaños de los efectos ya conocidos por los predictores de Utaut (Venkatesh; Thong; Xu, 2012; Taiwo; Downe, 2013), un error tipo 1, $\alpha$, de 0,05 y una potencia estadística (1- $\beta$ ) de 0,80. El meta-análisis de Taiwo y Downe (2013) encontró correlaciones (Zr) pequeñas o medianas entre la Expectativa de rendimiento y la intención de uso $(\mathrm{Zr}=0,54)$, la Expectativa de esfuerzo y la intención de uso $(\mathrm{Zr}=0,44)$ y la Influencia social y la intención de uso $(\mathrm{Zr}=0,42)$. Usando esta referencia y para detectar efectos medianos $\left(f^{2}=0,15\right)$ (Cohen, 1992; Faul et al., 2007) en nuestra regresión lineal múltiple con tres predictores, obtuvimos que la muestra mínima necesaria es de 43 casos.

Para obtener esta muestra se invitó a 300 editores de las 20 principales revistas de ciencias sociales en Google Scholar Metrics, que forman parte de las siguientes áreas:

- Antropología;

- Negocios, economía y administración;

- Comunicación;

- Ciencias sociales;

- Educación;

- Geografía y estudios urbanos;

- Derecho;

- Biblioteca y ciencias de la información;

- Ciencia política, administración y relaciones internacionales;

- Psicología;

- Sociología;

- Obras sociales y servicios;

- Pruebas académicas y psicológicas;

- Estudios africanos e historia;

- multidisciplinar.

Después de la invitación en octubre de 2016, 56 editores respondieron correctamente al cuestionario, una tasa de respuesta del $20 \%$.

\subsection{Medidas utilizadas}

Los indicadores utilizados se componían de varios items para cada variable:

- Expectativa de rendimiento ( 4 items, $\alpha_{c}=0,87$ );

- Expectativa de esfuerzo ${ }^{1}\left(4\right.$ items, $\alpha_{c}=0,87$ ) (medida del 1 al 5 , donde el 5 es más facilidad);

- Influencia social (3 items, $\alpha_{c}=0,83$ ).

Para realizar la medición se utilizó una escala de Likert de 5 puntos, desde el 1 (completamente en desacuerdo) hasta el 5 (completamente de acuerdo) (tabla 1). Las mediciones de la intención de uso diario, frecuente y futuro, se hicieron para cada red social, por el mismo sistema, encontrando en cada caso una fiabilidad de:

- Twitter: $\alpha_{c}=0,94$

- Facebook: $\alpha_{c}=0,92$

- ResearchGate: $\alpha=0,75$

- Academia: $\alpha_{c}=0,58$

- LinkedIn: $\alpha_{c}=0,72$. 
La frecuencia de uso se midió con la misma escala, desde nunca (1) hasta siempre (5). El uso real se midió con respuesta dicotómica (0, No adoptado; 1 , Sí adoptado). Además se consideraron variables sociodemográficas:

- edad;

- género (1=hombre, 2=mujer);

- nivel académico;

- estatus laboral;

- país de trabajo;

- temática editorial.

No hay estudios precedentes que exploren a los editores de revistas científicas desde una perspectiva teórica de adopción de innovaciones tecnológicas

El estadístico Alpha de Cronbach $\left(\alpha_{c}\right)$ se utilizó para determinar la fiabilidad por la consistencia interna, garantizando el mínimo requerido 0,70 (Cronbach, 1951).

\subsection{Análisis}

Se comenzó el análisis realizando una revisión de los datos después de codificarlos para detectar posibles errores. Los valores perdidos fueron estimados (Myers, 2011) usando regresiones ordinarias de mínimos cuadrados con 5 imputaciones. A continuación, con los datos depurados, se realizó un análisis estadístico inductivo-exploratorio que aportaba información del uso de las redes sociales. Para finalizar se llevó a cabo un análisis de mediación moderada para comprobar las hipótesis por medio del programa SPSS y la macro Process (Modelo 9) (Hayes, 2013), con 10.000 muestras de bootstrap (95\% e intervalos de confianza corregidos por sesgo). Este método está basado en el análisis de regresión lineal múltiple estimado por mínimos cuadrados ordinarios (OLS).

\section{Resultados}

\subsection{Análisis exploratorio de los datos}

Los resultados exploratorios obtenidos sobre la tasa de adopción de los medios sociales estudiados (RQ1), indican que la tasa promedio es del 38,21\% ( $M=1,88 \mathrm{DE}=0,63)$, lo que significa que la adopción es baja si se tienen en cuenta todas las redes en su conjunto. Pero el uso real de cada red a nivel individual varía, siendo las tasas de adopción:

- Twitter: 69,60\% (M=0,69 DE=0,46)

- Facebook: $57,14 \%(\mathrm{M}=0,57 \mathrm{DE}=0,49)$

- LinkedIn: 26,80\% (M=0,26 DE=0,44)

- ResearchGate: $21,40 \%(\mathrm{M}=0,21 \mathrm{DE}=0,41)$

- Academia: $16,10 \%(\mathrm{M}=0,16 \mathrm{DE}=0,37)$.

Las redes con mayor frecuencia de acceso en la escala de 1-5 son, de mayor a menor:

- Twitter: (M=2,66 DE=1,37)

- Facebook: (M=2,24 DE=1,30)

- ResearchGate: $(\mathrm{M}=1,63, \mathrm{DE}=1,04)$

- LinkedIn:(M=1,54 DE=0,83)

- Academia: (M=1,34 DE=0,63).

Tabla 1. Expectativa de rendimiento, Expectativa de esfuerzo e Influencia social.

\begin{tabular}{|l|c|c|}
\hline Expectativa de rendimiento & M & DE \\
\hline Usar las redes sociales aumenta el impacto de mi revista & 3,52 & 1,10 \\
\hline El uso de las redes sociales aumenta las posibilidades de mi revista de lograr cosas importantes para ella & 3,50 & 1,10 \\
\hline Encuentro los medios sociales útiles en mi revista & 3,30 & 1,18 \\
\hline El uso de las redes sociales ayuda a mi revista a lograr cosas más rápidamente & 2,97 & 1,09 \\
\hline Total & 3,32 & 0,95 \\
\hline Expectativa de esfuerzo & M & DE \\
\hline El personal de mi revista encuentra que las redes sociales son fáciles de usar & 3,54 & 1,01 \\
\hline Es fácil para el personal de mi revista llegar a ser hábil en las redes sociales & 3,51 & 1,03 \\
\hline Aprender a usar las redes sociales es fácil para el personal de mi revista & 3,48 \\
\hline La interacción del personal de mi revista con las redes sociales es clara y comprensible & 3,00 \\
\hline Total & 3,12 & 1,05 \\
\hline Influencia social & 3,41 & 0,87 \\
\hline $\begin{array}{l}\text { Las personas que influyen en el comportamiento de mi revista (editor, patrocinadores) piensan que debería usar las } \\
\text { redes sociales }\end{array}$ & $\mathbf{M}$ & $\mathbf{D E}$ \\
\hline Las personas que son importantes para mi revista, piensan que debería usar las redes sociales & 3,56 \\
\hline Las personas cuyas opiniones son valoradas por mi revista (autores, lectores) prefieren que use las redes sociales & 1,22 \\
\hline Total & 3,34 & 1,11 \\
\hline
\end{tabular}


Twitter y Facebook son por tanto las más usadas y las de acceso más frecuente.

La intención de uso (en una escala de 1 a 5) muestra también que Twitter ( $M=3,38 \mathrm{DE}=1,50)$ y Facebook ( $\mathrm{M}=2,82$ $\mathrm{DE}=1,55)$ son las preferentes, seguidas de ResearchGate $(\mathrm{M}=1,77 \mathrm{DE}=1,33)$, Academia $(\mathrm{M}=1,47 \mathrm{DE}=0,85)$ y por último LinkedIn ( $M=1,52 \mathrm{DE}=1,13)$. Tomando estos valores, la media se sitúa en 2,18 $(\mathrm{DE}=0,66)$.

Los datos obtenidos muestran que los editores tenían una Expectativa de rendimiento (EdR) en una escala de 1 a 5 hacia las redes sociales, con valores de 2,97 a 3,52, lo que implica que su percepción es positiva, siendo la media de 3,32 $(\mathrm{M}=3,32 \mathrm{DE}=0,95)$.

En cuanto a la Expectativa de esfuerzo (EdE) en la escala de 1 a 5, los valores van de 3,12 a 3,54, obteniendo una media de 3,41, lo que implica que su percepción es baja.

La Influencia social ha obtenido unos valores que van de 3,23 a 3,56, obteniendo una media de 3,38, lo que implica que su percepción es alta (tabla 1).

\subsection{Análisis de mediación moderada}

\subsubsection{Expectativa de rendimiento}

Para la variable independiente Expectativa de rendimiento (EdR) se ha realizado un análisis de mediación moderada en cada red social (figura 2).

Para Facebook obtenemos que el efecto directo de la Expectativa de rendimiento sobre el uso real no se ha mostrado significativo $(B=-1,07, p=0,07, I C=d e-2,24$ a $0,09)$. A pesar de ello existe un efecto condicional indirecto que delata esa relación (tabla 2) a través de la intención de uso (el efecto, con valores de 0,86 a 1,85, fue significativo para los 3 niveles de género moderador

La Expectativa de rendimiento es el factor predictivo más fuerte en la adopción de una tecnología, pero en este estudio no se confirma totalmente el modelo Utaut masculino y no significativo en la totalidad del género femenino). Este resultado sostiene $\mathrm{H} 1$, que postulaba que la Expectativa de rendimiento influencia la intención de uso y ésta, a su vez, influye de modo positivo en el uso real de las redes sociales, con su efecto moderado por la edad y el género, siendo más fuerte en los hombres jóvenes.

Para Twitter, el efecto directo de la Expectativa de rendimiento sobre el uso real no se mostró significativo ( $B=0,22$, $\mathrm{p}=0,71, \mathrm{IC}=-0,94$ a 1,38). Pero sí se observó esa relación a través de un efecto condicional indirecto (el efecto resultó significativo para los 4 niveles de la variable edad moderadora más altos en ambos géneros, con variación de 1,49 a 1,64), lo que apoya $\mathrm{H} 1$ y fue más fuerte en las mujeres de menor edad, lo que nos indica que existe moderación de la edad y el género, pero contrario a lo planteado en $\mathrm{H} 1$.

En ResearchGate no se aprecia que sea significativo el efecto directo de la Expectativa de rendimiento sobre el uso real $(B=-0,29 ; p=0,54$; $\mathrm{IC}=-1,22$ a 0,65$)$. Tampoco se produce relación de modo indirecto a través de la intención de uso (el efecto no se mostró significativo en edad ni género, variando de -0,52 a 0,93, siendo más fuerte en las mujeres mayores, en la dirección opuesta a lo planteado en $\mathrm{H} 1$ ).

Para Academia, no fue significativo el efecto directo que ejerce la Expectativa de rendimiento sobre el uso real $(B=-0,33, p=0,60, I C=-1,59$ a 0,93$)$. La relación tampoco se ha revelado a través de un efecto condicional indirecto de la intención de uso (los valores del efecto variaron de $-0,37$ a $-0,47$, no siendo sig-

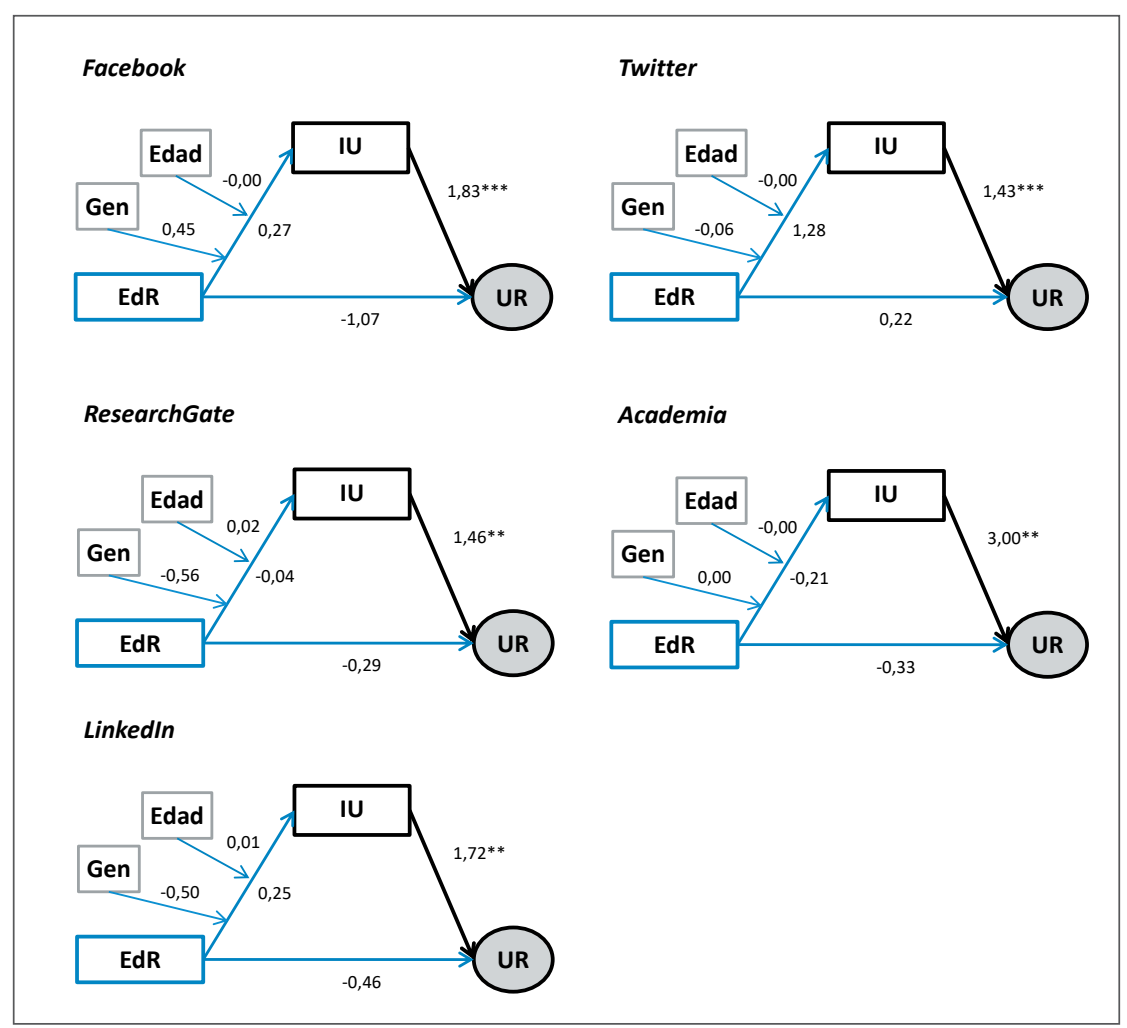

Figura 2. Expectativa de rendimiento. Coeficientes no normalizados de regresión (B) para cada modelo. $(*=\mathrm{P}<0,05 ; * *=\mathrm{P}<0,01 ; * * *=\mathrm{P}<0,001)$ 
nificativo para edad ni género, a pesar de ser más fuerte en hombres y mujeres en los tramos de mayor edad, de modo opuesto a lo reflejado en $\mathrm{H} 1$ ).

Para Linkedln tampoco fue significativo el efecto directo sobre el uso real de la Expectativa de rendimiento $(B=-0,46, p=0,27$, IC $=-1,29$ a 0,36). Igualmente, no se produce relación mediando la intención de uso (la variación del efecto fue de $-0,53$ a 0,74 , no siendo significativo para el moderador edad ni género, y mostrándose más fuerte en mujeres de los 3 niveles de edad, opuesto a H1) (tabla 2).

\subsubsection{Expectativa de esfuerzo}

Para la variable independiente Expectativa de esfuerzo (EdE), se ha realizado un análisis de mediación moderada para una de las redes sociales (figura 3).

Comenzando con Facebook, el

efecto directo de la Expectativa de esfuerzo sobre el uso real no fue significativo $(B=-0,44, p=0,32, I C=d e-1,30$ a 0,43$)$, ni hubo relación a través de la intención de uso mediante un efecto condicional indirecto (sus valores variaron de $-0,02$ a 0,48 , no siendo significativo para el moderador edad ni género, y mostrándose más fuerte en los hombres de más edad, en el sentido opuesto de género al planteado en $\mathrm{H} 2$ ).

Atendiendo a Twitter, no se mostró significativo el efecto directo de la Expectativa de esfuerzo sobre el uso real ( $B=-$ $0,314, p=0,55, I C=d e-1,35$ a 0,72 ). Esta relación tampoco se produjo a través de la intención de uso mediante un efecto condicional indirecto (los valores variaron de - 0,24 a 1,10, no siendo significativo para el moderador edad ni género, y mostrándose más fuerte en las mujeres más mayores, lo que apoya $\mathrm{H} 2$ ).

Para ResearchGate el efecto directo de la Expectativa de esfuerzo sobre el uso real no fue significativo $(B=-0,34, p=0,50$, $\mathrm{IC}=-1,33$ a 0,65$)$. No se encontró otra vía de relación mediante la intención de uso con un efecto no directo (los valores variaron de -0,20 a 1,08, no siendo significativo para el moderador edad ni género, pero mostrándose con más intensidad en mujeres más mayores, en línea con $\mathrm{H} 2$ ).

En Academia no se ha demostrado un efecto directo de la Expectativa de esfuerzo sobre el uso real $(B=-$ $0,57, p=0,39, I C=-0,72$ a 1,86$)$. De forma similar, no se ha producido un vínculo a través de la intención de uso mediante un efecto condicional indirecto (los valores variaban de -0,20 a 0,10, de manera que no fue significativo para el moderador edad ni género, siendo más fuerte en los hombres más mayores, lo que indica un sentido opuesto de género al planteado en $\mathrm{H} 2$ pero coincidiendo en edad).

Por último, en Linkedln no fue significativo un efecto directo de la Expectativa de esfuerzo sobre el uso real ( $B=0,65$, $p=0,20, I C=-0,35$ a 1,66). No se ha revelado tampoco conexión indirecta mediante la intención de uso (los valores del efecto variaron de $-0,73$ a 0,20 , y no fue significativo en el moderador edad ni género -excepto en hombres de 54 años, pero siendo más intenso en las mujeres de más edad, sosteniendo lo propuesto en $\mathrm{H} 2$ ).

\subsubsection{Influencia social}

Para la variable independiente Influencia social (IS), se ha realizado un análisis de mediación moderada en cada uno de los medios sociales (figura 4).

En el caso de Facebook, el efecto directo de la Influencia social sobre el uso real no fue significativo $(B=-0,47, p=0,27$, $\mathrm{IC}=-1,30$ a 0,36). A pesar de ello, a través de la intención de uso se genera un efecto condicional indirecto (los valores variaron de 0,75 a 0,98 y sólo fueron significativos para el nivel intermedio de edad en hombres), siendo más fuerte en los hombres más jóvenes, lo que muestra un sentido opuesto al planteado en H3 (que indicaba que el efecto de la Influencia 
social sobre la intención de uso es moderado significativamente por el género y la edad, de tal manera que el efecto será más fuerte en las mujeres y en las personas de más edad).

En Twitter, de un modo directo no fue significativo el efecto de la Influencia social sobre el uso real $(B=-0,59, p=0,23, I C=-0,37$ a 1,56$)$. La relación en cambio, sí se aprecia a través de la intención de uso, con un efecto condicional indirecto (los valores variaron de 0,50 a 1,23 y sólo fueron significativos para el nivel intermedio de edad en hombres), siendo más fuerte en los hombres y en los más jóvenes, en sentido opuesto a H3.

ResearchGate no muestra un efecto directo significativo de la Influencia social sobre el uso real $(B=-0,04, p=0,92, I C=-0,87$ a 0,79$)$. Además, no se aprecia que la intención de uso tenga un efecto condicional indirecto (varió de $-0,76$ a 0,27 , no fue significativo para edad ni género, aunque mostraba más intensidad en los hombres y en los más jóvenes, en sentido opuesto a H3).

En Academia no se muestra significancia en el efecto directo de la Influencia social sobre el uso real $(B=0,90, p=0,14, I C=-$ 0,29 a 2,10). De igual forma, no hubo relación mediante un efecto condicional indirecto a través de la intención de uso (el valor variaba de $-1,25$ a 0,13, no fue significativo para ninguno de los niveles de edad ni género, pero era más intenso en los hombres y en los más jóvenes, en sentido opuesto a $\mathrm{H3}$ ).

En LinkedIn no fue significativo el efecto directo de la Influencia social sobre el uso real $(B=-0,02, p=0,96, I C=-0,82$ a 0,78$)$. De igual modo no se aprecia un efecto condicional indirecto reseñable a través de la intención de uso (varió de -1,09 a 0,25 , pero no fue significativo para ninguno de los niveles de edad ni género, mostrando más intensidad en los hombres y en los más jóvenes, lo que es opuesto a H3).

\section{Discusión y conclusión}

Hay que considerar en primer lugar que los tipos de redes sociales estudiadas son diversos, puesto que se han incluido redes sociales generalistas (Twitter y Facebook), académicas (ResearchGate y Academia), y profesionales (Linkedln), para estudiar el uso que hacen de ellas los editores de revistas de ciencias sociales específicamente. A pesar de que las dos redes generalistas son las más extendidas, su uso por investigadores o con fines académicos genera escepticismo (Rúas-Araújo; Campos-Freire; Puentes-Rivera, 2016). En este caso se trata la utilización de estas de redes sociales por revistas de ciencias sociales, cuya finalidad es la difusión de su contenido y trabajo diario.

En atención a los datos obtenidos, la tasa de adopción real de las redes sociales (RQ1) es baja (38,21\%) cuando revisamos el conjunto de las redes analizadas (Facebook, Twitter, ResearchGate, Academia y LinkedIn).

Pero si analizamos la tasa de adopción individual apreciamos que varía, siendo la de mayor adopción Twitter (69,60\%), seguida de Facebook (57,14\%), LinkedIn (26,80\%), ResearchGate (21,40\%) y Academia (16,10\%). En la frecuencia de acceso se colocan en primer lugar Twitter y Facebook, seguidas de ResearchGate, Linkedln y Academia. La intención de uso medio es mayor en Twitter y Facebook, seguidas de ResearchGate, LinkedIn y Academia. No se aprecia en ninguno de los casos que exista un efecto directo de la Expectativa de rendimiento sobre el uso real, sino que en alguna de las relaciones el efecto es mediado por la intención de uso. A su vez, el uso real recibe un efecto indirecto de la Expectativa de rendimiento que está moderado en algunos casos por el género y la edad, según propone el modelo Utaut (Venkatesh et al., 2003; Venkatesh; Thong; $\mathrm{Xu}, 2012)$, generando un efecto condicional indirecto.
Los efectos de la Expectativa de rendimiento sobre el uso real a través de la intención de uso de medios sociales por los editores de las revistas sólo son significativos en el caso de Facebook y Twitter 
Tabla 2. Efecto condicional indirecto de la EdR, EdE e IS en el uso real a través de la intención de uso, moderada por edad y género.

\begin{tabular}{|c|c|c|c|c|c|c|c|c|c|c|c|c|c|c|}
\hline \multirow{2}{*}{$\begin{array}{l}\text { Redes } \\
\text { socia- } \\
\text { les }\end{array}$} & \multirow[b]{2}{*}{ Edad } & \multirow[b]{2}{*}{$\begin{array}{l}\text { Gé- } \\
\text { nero }\end{array}$} & \multicolumn{4}{|c|}{ Expectativa de rendimiento } & \multicolumn{4}{|c|}{ Expectativa de esfuerzo } & \multicolumn{4}{|c|}{ Influencia social } \\
\hline & & & Efecto & $\begin{array}{c}\text { BOOT } \\
\text { SE }\end{array}$ & $\begin{array}{c}\text { BOOT } \\
\text { LLCI }\end{array}$ & $\begin{array}{l}\text { BOOT } \\
\text { ULCI }\end{array}$ & Efecto & $\begin{array}{c}\text { BOOT } \\
\text { SE }\end{array}$ & $\begin{array}{c}\text { BOOT } \\
\text { LLCI }\end{array}$ & $\begin{array}{l}\text { BOOT } \\
\text { ULCI }\end{array}$ & Efecto & $\begin{array}{c}\text { BOOT } \\
\text { SE }\end{array}$ & $\begin{array}{l}\text { BOOT } \\
\text { LLCI }\end{array}$ & $\begin{array}{l}\text { BOOT } \\
\text { ULCI }\end{array}$ \\
\hline \multirow{6}{*}{$F B$} & 42 & $\mathrm{~F}$ & 1,02 & 1,44 & $-1,02$ & 3,89 & $-0,02$ & 0,90 & $-1,55$ & 2,03 & 0,83 & 0,98 & $-0,92$ & 2,85 \\
\hline & 42 & M & 1,85 & 1,28 & 0,32 & 3,34 & 0,31 & 0,97 & $-1,74$ & 2,08 & 0,98 & 0,89 & $-0,38$ & 2,88 \\
\hline & 54 & $\mathrm{~F}$ & 0,94 & 1,47 & $-1,89$ & 3,49 & 0,06 & 0,79 & $-1,40$ & 1,79 & 0,79 & 1,07 & $-1,36$ & 2,73 \\
\hline & 54 & M & 1,77 & 1,05 & 0,75 & 3,14 & 0,40 & 0,64 & $-0,70$ & 1,77 & 0,95 & 0,67 & 0,06 & 2,42 \\
\hline & 65 & $\mathrm{~F}$ & 0,86 & 1,69 & $-2,65$ & 3,76 & 0,15 & 0,95 & $-1,85$ & 1,99 & 0,75 & 1,29 & $-1,98$ & 3,07 \\
\hline & 65 & $M$ & 1,69 & 1,08 & 0,44 & 3,89 & 0,48 & 0,61 & $-0,48$ & 1,98 & 0,91 & 0,67 & $-0,15$ & 2,23 \\
\hline \multirow{6}{*}{$T W$} & 42 & $\mathrm{~F}$ & 1,64 & 1,88 & $-0,22$ & 3,89 & 0,26 & 1,24 & $-1,30$ & 2,86 & 0,96 & 1,38 & $-0,57$ & 4,24 \\
\hline & 42 & $M$ & 1,55 & 1,56 & $-0,15$ & 3,39 & $-0,25$ & 1,47 & $-4,48$ & 1,37 & 1,23 & 1,48 & $-0,24$ & 3,96 \\
\hline & 54 & $\mathrm{~F}$ & 1,61 & 1,82 & 0,00 & 4,21 & 0,68 & 1,20 & $-1,41$ & 2,47 & 0,73 & 1,29 & $-0,84$ & 4,18 \\
\hline & 54 & $M$ & 1,52 & 1,43 & 0,07 & 3,45 & 0,17 & 1,05 & $-1,90$ & 1,64 & 1,00 & 1,16 & 0,00 & 3,44 \\
\hline & 65 & $\mathrm{~F}$ & 1,58 & 1,85 & 0,05 & 5,31 & 1,10 & 1,74 & $-1,39$ & 4,46 & 0,50 & 1,47 & $-1,72$ & 3,78 \\
\hline & 65 & $M$ & 1,49 & 1,41 & 0,41 & 5,43 & 0,59 & 1,31 & $-0,71$ & 4,23 & 0,77 & 1,11 & $-0,43$ & 3,41 \\
\hline \multirow{6}{*}{$R G$} & 42 & $\mathrm{~F}$ & 0,29 & 1,54 & $-1,24$ & 4,39 & 0,42 & 1,14 & $-1,16$ & 2,81 & $-0,67$ & 2,06 & $-3,52$ & 0,30 \\
\hline & 42 & $M$ & $-0,52$ & 1,41 & $-3,27$ & 0,24 & $-0,20$ & 1,14 & $-2,74$ & 0,87 & 0,27 & 0,94 & $-0,49$ & 2,78 \\
\hline & 54 & $\mathrm{~F}$ & 0,61 & 2,03 & $-0,98$ & 5,27 & 0,75 & 1,56 & $-0,90$ & 4,00 & $-0,72$ & 2,21 & $-4,14$ & 0,44 \\
\hline & 54 & $M$ & $-0,20$ & 0,75 & $-1,61$ & 0,45 & 0,13 & 0,75 & $-0,97$ & 1,65 & 0,23 & 0,72 & $-0,24$ & 2,22 \\
\hline & 65 & $\mathrm{~F}$ & 0,93 & 2,69 & $-1,08$ & 6,65 & 1,08 & 2,28 & $-0,97$ & 5,65 & $-0,76$ & 2,52 & $-5,53$ & 0,84 \\
\hline & 65 & $M$ & 0,12 & 0,69 & $-0,71$ & 2,04 & 0,46 & 1,21 & $-0,82$ & 2,85 & 0,18 & 1,01 & $-0,99$ & 2,36 \\
\hline \multirow{6}{*}{$A C$} & 42 & $\mathrm{~F}$ & $-0,47$ & 1,51 & $-2,50$ & 2,36 & $-0,20$ & 1,66 & $-2,13$ & 4,51 & $-0,93$ & 3,05 & $-7,89$ & 1,87 \\
\hline & 42 & $M$ & $-0,47$ & 1,59 & $-3,79$ & 0,73 & $-0,09$ & 1,72 & $-3,70$ & 2,45 & 0,13 & 1,27 & $-3,20$ & 2,36 \\
\hline & 54 & $\mathrm{~F}$ & $-0,42$ & 1,55 & $-3,09$ & 2,13 & $-0,10$ & 1,75 & $-2,38$ & 3,62 & $-1,09$ & 3,30 & $-9,10$ & 1,78 \\
\hline & 54 & $M$ & $-0,41$ & 1,02 & $-2,23$ & 0,50 & 0,01 & 1,33 & $-2,07$ & 2,89 & $-0,03$ & 0,97 & $-1,97$ & 2,20 \\
\hline & 65 & $\mathrm{~F}$ & $-0,37$ & 1,98 & $-4,10$ & 3,04 & 0,00 & 2,43 & $-4,05$ & 3,65 & $-1,25$ & 3,80 & $-10,84$ & 2,64 \\
\hline & 65 & $M$ & $-0,37$ & 0,94 & $-1,81$ & 1,45 & 0,11 & 1,77 & $-2,68$ & 3,97 & $-0,19$ & 1,48 & $-2,78$ & 4,05 \\
\hline \multirow{6}{*}{$L K$} & 42 & $F$ & 0,33 & 0,74 & $-0,95$ & 1,78 & 0,11 & 0,71 & $-1,14$ & 1,66 & $-0,37$ & 1,45 & $-3,86$ & 0,84 \\
\hline & 42 & $M$ & $-0,53$ & 0,77 & $-2,79$ & 0,09 & $-0,74$ & 0,77 & $-2,86$ & 0,32 & 0,25 & 0,59 & $-0,59$ & 1,26 \\
\hline & 54 & $\mathrm{~F}$ & 0,53 & 0,86 & $-1,20$ & 2,00 & 0,16 & 0,80 & $-1,58$ & 1,44 & $-0,73$ & 1,83 & $-5,10$ & 0,56 \\
\hline & 54 & $M$ & $-0,32$ & 0,53 & $-1,63$ & 0,18 & $-0,69$ & 0,55 & $-2,05$ & $-0,05$ & $-0,11$ & 0,30 & $-0,68$ & 0,43 \\
\hline & 65 & $\mathrm{~F}$ & 0,74 & 1,10 & $-1,45$ & 2,74 & 0,20 & 1,06 & $-2,38$ & 1,55 & $-1,09$ & 2,24 & $-6,31$ & 0,49 \\
\hline & 65 & $M$ & $-0,12$ & 0,53 & $-0,99$ & 0,92 & $-0,65$ & 0,59 & $-1,68$ & 0,29 & $-0,47$ & 0,50 & $-1,38$ & 0,25 \\
\hline
\end{tabular}

Los resultados obtenidos sobre este efecto condicional indirecto ejercido por la variable Expectativa de rendimiento, a través de la intención de uso, sobre el uso real de redes sociales por los editores de las revistas (H1), sólo son significativos en el caso de Facebook y Twitter. Esto supone que si un editor considera que la adopción de esos medios sociales puede mejorar el rendimiento de su revista en el futuro, aumenta su intención de usarlo y por lo tanto la probabilidad de uso real.

Facebook muestra un efecto indirecto condicional más fuerte en los hombres jóvenes, apoyando H1. Twitter muestra un efecto indirecto más fuerte en las mujeres jóvenes, en sentido opuesto a H1. Los resultados de la mediación no son significativos en ResearchGate y LinkedIn, donde el efecto indirecto fue más intenso en mujeres de mayor edad, mientras que en Academia no se aprecia diferencia de género y el efecto aumenta con la edad.

No hay suficiente evidencia empírica de que la variable Expectativa de rendimiento tenga influencia en el uso real de estas tres redes sociales a través de la variable intención de uso, ni que la edad o el género moderen el tamaño en ningún sentido.

Por lo tanto, podemos indicar que la Expectativa de rendimiento es un importante factor de predicción de uso en determinadas redes sociales con medidas de coeficientes no normalizados de hasta 3,00. También conocida como utilidad percibida (Davis, 1989; Davis; Bagozzi; Warshaw, 1992) es determinante en la adopción de tecnologías, indicando que la decisión de adoptar o no una tecnología está condicionada por el convencimiento individual. 
Revisando $\mathrm{H} 2$, los resultados del análisis del efecto indirecto negativo ejercido por la Expectativa de esfuerzo a través de la intención de uso, sobre el uso real de redes sociales por los editores de las revistas, no son significativos. Si los editores valoran el esfuerzo que supone la adopción de determinadas redes sociales baja la probabilidad de su uso real. No se aprecia en ninguno de los casos un efecto directo o indirecto de la Expectativa de esfuerzo sobre el uso real, según propone Utaut (Venkatesh et al., 2003; Venkatesh; Thong; Xu, 2012). En Twitter, ResearchGate y LinkedIn el efecto de interacción fue más fuerte en las mujeres mayores. En Facebook y Academia el efecto es más intenso en hombres mayores. Por tanto, la Expectativa de esfuerzo no se puede considerar un predictor de uso negativo de los medios sociales con medidas de coeficientes no normalizados hasta 3,28 , y no podemos demostrar empíricamente el efecto condicional indirecto, ni una moderación de género o edad con una dirección clara y aplicable a todas las redes sociales estudiadas.

Por último, valorando H3, los resultados confirman un efecto condicional indirecto positivo ejercido por la Influencia social a través de la intención de uso, sobre el uso real de redes sociales por los editores, pero sólo en caso de Facebook y Twitter. Esto supone que cuando los editores se sienten influenciados positivamente en su entorno social, colaboradores o amigos, aumenta la probabilidad de que adopten dichos medios sociales. No apreciamos en ningún caso un efecto directo significativo de la Influencia social sobre el uso real. El efecto de la Influencia social sobre el uso real, se modera con las variables de género y edad según propone el modelo Utaut (Venkatesh et al., 2003; Venkatesh; Thong; Xu, 2012), y muestra un efecto más intenso en mujeres jóvenes, opuesto a H3. Podemos considerar que la Influencia social es predictor del uso de Facebook y Twitter, con medidas de coeficientes no normalizados hasta 3,66, y que el género y la edad pueden ser moderadores más fuertes en mujeres jóvenes.

Podemos valorar las implicaciones teóricas o prácticas de estos resultados. Para empezar, en línea con los estudios previos existentes, se confirma que el factor predictivo más fuerte en la adopción de una tecnología es la Expectativa de rendimiento. A pesar de ello, en esta investigación concreta se ha demostrado un desacuerdo con el modelo Utaut y Utaut2 en cuanto a la influencia que las variables género y edad ejercen en el uso real de una tecnología, porque no son en todos los casos moderadoras de la relación entre los factores explicativos y el uso real. Tampoco apreciamos que la variable Expectativa de esfuerzo sea un predictor significativo en ninguno de los casos analizados. Por último, atendiendo a las implicaciones prácticas, este estudio puede servir de base para la generación de políticas de adopción de TIC por investigadores, considerando la utilidad que se per-

La Influencia social es predictor del uso de Facebook y Twitter, y el género y la edad pueden ser moderadores más fuertes en mujeres jóvenes

cibe y los beneficios obtenidos. Hay algunas limitaciones que también hay que reconocer. Estamos considerando un uso profesional de los medios sociales aplicados en revistas científicas de ciencias sociales, por lo que investigaciones futuras se pueden encaminar a incluir los condicionantes existentes en este entorno como son la formación y experiencia previa, las condiciones facilitadas y las competencias tecnológicas individuales. También se pueden estudiar los usos concretos que las revistas aplican en cada medio social teniendo en cuenta los perfiles de uso y consumo concreto de cada tipo de red y ampliar la investigación a otros medios sociales según el objetivo de comunicación de la propia revista.

\section{Nota}

1. La Expectativa de esfuerzo se mide como el nivel de facilidad percibida, de modo contrario a la teoría del modelo UTAUT, por lo que la correlación esperada es positiva. Números altos suponen baja Expectativa de esfuerzo y número bajos suponen alta Expectativa de esfuerzo.

\section{Referencias}

Acord, Sophia-Krzys; Harley, Diane (2013). "Credit, time, and personality: The human challenges to sharing scholarly work using Web 2.0". New media \& society, v. 15, n. 3, pp. 379-397.

https://doi.org/10.1177/1461444812465140

Arcila-Calderón, Carlos; Calderín-Cruz, Mabel; Aguaded, Ignacio (2015). “Adoption of ICTs by communication researchers for scientific diffusion and data analysis". El profesional de la información, v. 24, n. 5, p. 526-536. https://doi.org/10.3145/epi.2015.sep.03

Arcila-Calderón, Carlos; Piñuel-Raigada, José-Luis; Calderín-Cruz, Mabel (2013). "The e-research on media \& communications: Attitudes, tools and practices in Latin America researchers". Comunicar, v. 20, n. 40, pp. 111-118.

https://doi.org/10.3916/C40-2013-03-01

Barjak, Franz; Lane, Julia; Poschen, Meik; Procter, Rob; Robinson, Simon; Wiegand, Gordon (2010). "E-infraestructure adoption in the social sciences and humanities". Information, communication \& society, v. 13, n. 5, pp. 635-651. https://doi.org/10.1080/13691180903095849

Bellón-Rodríguez, Ana; Sixto-García, José (2011). “Aplicación y uso de la web 2.0 y de las redes sociales en la comunicación científica especializada: del marketing viral al usuario activo". Anagramas. Rumbos y sentidos de la comunicación, v. 9 , n. 18 , pp. 61-70.

https://doi.org/10.22395/angr.v9n18a4 
Borgman, Christine L. (2007). Scholarship in the digital age: Information, infrastructure, and the Internet. The MIT Press, ISBN: 9780262026192

Briceño, Ysabel (2014). "Saber y medios: hacia un modo emergente de la comunicación de la ciencia”. Bitácora-e. Revista electrónica latinoamericana de estudios sociales, históricos y culturales de la ciencia y la tecnología, v. 1.

http://www.saber.ula.ve/handle/123456789/38746

Briceño, Ysabel; Arcila-Calderón, Carlos; Said-Hung, Elías (2012). “Colaboración y comunicación científica en la comunidad latinoamericana de físicos de altas energías". E-colabora. Revista de ciencia, educación, innovación y cultura apoyadas por redes de tecnología avanzada, v. 2, n. 4, pp. 131-144.

Bruns, Axel; Liang, Yuxian-Eugene (2012). "Tools and methods for capturing Twitter data during natural disasters". First Monday, v. 17, n. 4.

https://doi.org/10.5210/fm.v17i4.3937

Campos-Freire, Francisco; Rúas-Araújo, José (2016). “Uso de las redes sociales digitales profesionales y científicas: el caso de las 3 universidades gallegas". El profesional de la información, v. 25, n. 3, p. 431-440.

https://doi.org/10.3145/epi.2016.may.13

Campos-Freire, Francisco; Valencia, Andrea (2015). "Managing academic profiles on scientific social networks". En: Rocha, Álvaro; Correia, Ana-María; Costanzo, Sandra; Reis, Luis-Paulo (eds.). New contributions in information sytems and technologies, v. 353 , pp. 265-273. ISBN: 9783319164854

https://doi.org/10.1007/978-3-319-16486-1_27

Castillo-Esparcia, Antonio (2012). "Investigación e investigadores. Las revistas científicas como instrumento de comunicación". Vivat Academia. Revista de comunicación, v. 14, n. 117, pp. 1002-1017.

https://doi.org/10.15178/va.2011.117E.1002-1017

Cohen, Jacob (1992). "Statistical power analysis". Current directions in psychological science, v. 1, n. 3, pp. 98-101.

https://doi.org/10.1111/1467-8721.ep10768783

Cronbach, Lee J. (1951). “Coefficient alpha and the internal structure of tests". Psychometrika, v. 16, n. 3, pp. $297-334$. https://doi.org/10.1007/BF02310555

Cummings, Jonathon N.; Kiesler, Sara (2005). "Collaborative research across disciplinary and organizational boundaries". Social studies of science, v. 35, n. 5, pp. 703-722.

https://doi.org/10.1177/0306312705055535

Davis, Fred D. (1989). "Perceived usefulness, perceived ease of use, and user acceptance of information technology". MIS quarterly, v. 13, n. 3, p. 319-340.

https://doi.org/10.2307/249008

Davis, Fred D.; Bagozzi, Richard P.; Warshaw, Paul R. (1992). "Extrinsic and intrinsic motivation to use computers in the workplace". Journal of applied social psychology, v. 22, n. 14, pp. 1111-1132.

https://doi.org/10.1111/j.1559-1816.1992.tb00945.x

Direito-Rebollal, Sabela; Campos-Freire, Francisco (2016). "Altmetrics: A measure of scientific impact on social networks". En: $11^{\text {th }}$ Iberian conf on information systems and technologies (Cisti), pp. 1-5.

https://doi.org/10.1109/CISTI.2016.7521616

Dutton, William H. (2010). "Reconfiguring access in research: information, expertise and experience". En: Dutton, WiIliam H.; Jeffreys, Paul W. World wide research: reshaping the sciences and humanities, pp. 21-39. MIT Press Scholarship Online. ISBN: 9780262014397

Dutton, William H.; Meyer, Eric T. (2009). "Experience with new tools and infrastructures of research: An exploratory study of distance from, and attitudes toward, e-research". Prometheus, v. 27, n. 3, pp. 223-238.

https://doi.org/10.1080/08109020903127802

Faul, Franz; Erdfelder, Edgar; Buchner, Axel; Lang, Albert-Georg (2009). "Statistical power analyses using G*Power 3.1: Tests for correlation and regression analyses". Behavior research methods, v. 41, n. 4, pp. 1149-1160.

https://doi.org/10.3758/BRM.41.4.1149

Faul, Franz; Erdfelder, Edgar; Lang, Albert-Georg; Buchner, Axel (2007). "G*Power 3: A flexible statistical power analysis program for the social, behavioral, and biomedical sciences". Behavior research methods, v. 39, n. 2, pp. $175-191$.

https://doi.org/10.3758/BF03193146

García-Peñalvo, Francisco-José (2017). Marco para la ciencia abierta. Seminario.

https://doi.org/10.5281/zenodo.1069582

Gentil-Beccot, Anne; Mele, Salvatore; Holtkamp, Annette; O’Connell, Heath B.; Brooks, Travis C. (2009). “Information 
resources in high-energy physics: Surveying the present landscape and charting the future course". Journal of the Association for Information Science and Technology, v. 60, n. 1, pp. 150-160.

https://doi.org/10.1002/asi.20944

Gibbons, Michael; Limoges, Camille; Nowotny, Helga; Schwartzman, Simon; Scott, Peter; Trow, Martin (1994). The new production of knowledge: The dynamics of science and research in contemporary societies. SAGE Publications. ISBN: 978 0803977945

Hara, Noriko; Solomon, Paul; Kim, Seung-Lye; Sonnenwald, Diane H. (2003). "An emerging view of scientific collaboration: Scientists' perspectives on collaboration and factors that impact collaboration". Journal of the Association for Information Science and Technology, v. 54, n. 10, pp. 952-965.

https://doi.org/10.1002/asi.10291

Hayes, Andrew F. (2013). Introduction to mediation, moderation, and conditional process analysis. New York, NY: Guilford. ISBN: 9781609182304

Liao, Chien-Hsiang (2011). "How to improve research quality? Examining the impacts of collaboration intensity and member diversity in collaboration networks". Scientometrics, v. 86, n. 3, pp. 747-761.

https://doi.org/10.1007/s11192-010-0309-2

Meyer, Eric T.; Dutton, William H. (2009). "Top-down e-infrastructure meets bottom-up research innovation: The social shaping of e-research". Prometheus, v. 27, n. 3, pp. 239-250.

https://doi.org/10.1080/08109020903127810

Murthy, Dhiraj; Lewis, Jeremiah P. (2015). "Social media, collaboration, and scientific organizations". American behavioral scientist, v. 59, n. 1, pp. 149-171.

https://doi.org/10.1177/0002764214540504

Myers, Teresa A. (2011). "Goodbye, listwise deletion: Presenting hot deck imputation as an easy and effective tool for handling missing data". Communication methods and measures, v. 5, n. 4, pp. 297-310.

https://doi.org/10.1080/19312458.2011.624490

Neylon, Cameron; Wu, Shirley (2008). “Open science: Tools, approaches and implications”. En: Biocomputing 2009, pp. 540-544.

https://doi.org/10.1142/9789812836939_0051

Oller-Alonso, Martín; Segarra-Saavedra, Jesús; Plaza-Nogueira, Alberto (2012). "La presencia de las revistas científicas de ciencias sociales en los social media: de la Web 1.0 a la 2.0". Index.comunicación: Revista científica en el ámbito de la comunicación aplicada, v. 2, n. 1, pp. 49-68.

http://journals.sfu.ca/indexcomunicacion/index.php/indexcomunicacion/article/view/27/367

Ponte, Diego; Simon, Judith (2011). "Scholarly communication 2.0: Exploring researchers' opinions on web 2.0 for scientific knowledge creation, evaluation and dissemination". Serials review, v. 37, n. 3, pp. 149-156.

https://doi.org/10.1080/00987913.2011.10765376

Procter, Rob; Voss, Alex; Asgari-Targhi, Marzieh (2013). "Fostering the human infrastructure of e-research". Information, communication \& society, v. 16, n. 10, pp. 1668-1691.

https://doi.org/10.1080/1369118X.2012.715667

Procter, Rob; Williams, Robin; Stewart, James; Poschen, Meik; Snee, Helene; Voss, Alex; Asgari-Targhi, Marzieh (2010). "Adoption and use of Web 2.0 in scholarly communications". Philosophical transactions of the Royal Society A: Mathematical, physical, and engineering sciences, v. 368, n. 1926, pp. 4039-56.

https://doi.org/10.1098/rsta.2010.0155

Quinlan, Stephen; Gummer, Tobias; Roßmann, Joss; Wolf, Christof (2017). “'Show me the money and the party!' variation in Facebook and Twitter adoption by politicians". Information, communication \& society, v. 21, n. 8, pp. 1-19. https://doi.org/10.1080/1369118X.2017.1301521

Rigby, John; Edler, Jakob (2005). "Peering inside research networks: Some observations on the effect of the intensity of collaboration on the variability of research quality". Research policy, v. 34, n. 6, pp. 784-794.

https://doi.org/10.1016/j.respol.2005.02.004

Rúas-Araújo, José; Campos-Freire, Francisco; Puentes-Rivera, Iván (2016). “Utilización y valoración de las redes sociales generalistas y buscadores bibliográficos en las universidades gallegas". Revista latina de comunicación social, n. 71, pp. 1187-1207. https://doi.org/10.4185/RLCS-2016-1141

Santana-Arroyo, Sonia (2011). "Redes de intercambio de información científica y académica entre los profesionales, en el contexto de la Web 2.0". Revista cubana de información en ciencias de la salud, v. 21, n. 3.

http://scielo.sld.cu/scielo.php?pid=S1024-94352010000300006\&script=sci_abstract 
Schiermeier, Quirin (2017). "Science publishers try new tack to combat unauthorized paper sharing". Nature, v. 545, n. 7653, pp. 145-146.

https://doi.org/10.1038/545145a

Stewart, James (2007). "Local experts in the domestication of information and communication technologies". Information, communication \& society, v. 10, n. 4, pp. 547-569.

https://doi.org/10.1080/13691180701560093

Taiwo, Ayankunle; Downe, Alan (2013). "The theory of user acceptance and use of technology (Utaut): A meta-analytic review of empirical findings". Journal of theoretical and applied information technology, v. 49, n. 1, pp. 48-58.

http://www.jatit.org/volumes/Vol49No1/7Vol49No1.pdf

Venkatesh, Viswanath; Morris, Michael G.; Davis, Gordon B.; Davis, Fred D. (2003). "User acceptance of information technology: Toward a unified view". MIS quarterly, v. 27, n. 3, pp. 425-478.

https://doi.org/10.2307/30036540

Venkatesh, Viswanath; Thong, James Y. L.; Xu, Xin (2012). “Consumer acceptance and use of information technology: Extending the unified theory of acceptance and use technology". MIS quarterly, v. 36, n. 1, pp. 157-172.

Voss, Alex; Asgari-Targhi, Marzieh; Procter, Rob; Fergusson, David (2010). "Adoption of e-infrastructure services: configurations of practice". Philosophical transactions. Series A, Mathematical, physical, and engineering sciences, v. $368, \mathrm{n}$. 1926, pp. 4161-4176.

https://doi.org/10.1098/rsta.2010.0162

Waldrop, Mitchell (2008). "Science 2.0. Is open access science the future? Is posting raw results online, for all to see, a great tool or a great risk?". Scientific American, v. 298, n. 5, pp. 68-73.

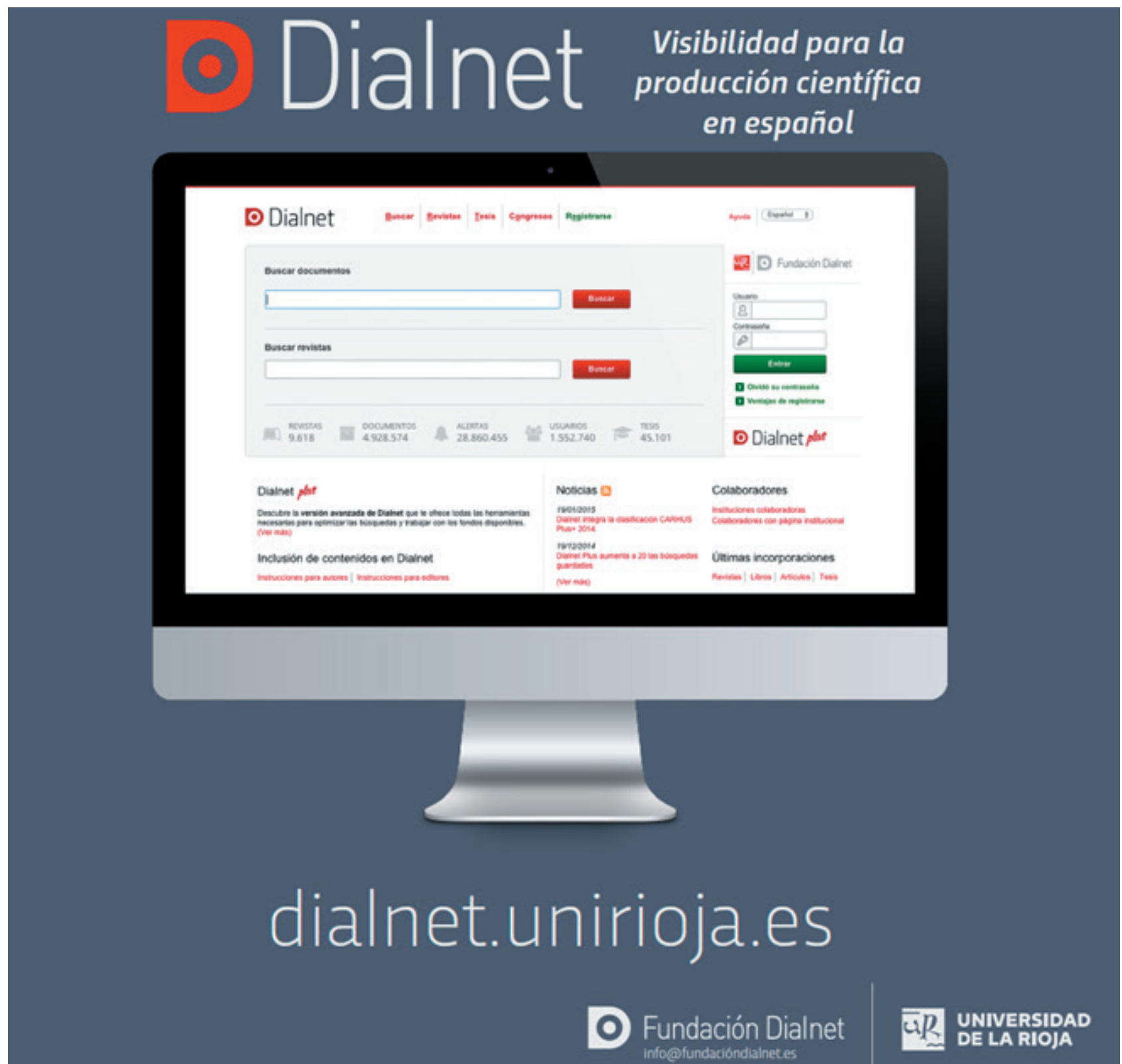

